\title{
Statistical Methodology Used In Analyses of Data From DOE Experimental Animal Studies
}

E.S. Gilbert

W.C. Griffith

B.A. CarnesI

July 1995

Prepared for the U.S. Department of Energy under Contract DE-AC06-76RLO 1830

Pacific Northwest Laboratory Operated for the U.S. Department of Energy by Battelle Memorial Institute 


\title{
DISCLAIMER
}

This report was prepared as an account of work sponsored by an agency of the United States Government. Neither the United States Government nor any agency thereof, nor Battelle Mernorial Institute nor any of their employees, makes any warranty, express or implied, or assumes any legal liability or responsibility for the accuracy, completeness, or usefulness of any information, apparatus, product, or process disclosed, or represents that its use would not infringe privately owned rights. Reference herein to any specific commercial product, process, or service by trade name, trademark, manufacturer, or otherwise does not necessárily constitute or imply its endorsement, recommendation, or favoring by the United States Government or any agency thereof, or Battelle Memorial Institute. The views and opinions of authors expressed herein do riot necessarily state or reflect those of the United States Government or any agency thereof.

\author{
PACIFIC NORTHWEST LABORATORY \\ operated by \\ BATTELLE MEMORIAL INSTITUTE \\ for the \\ UNITED STATES DEPARTMENT OF ENERGY \\ under Contract DE-AC06-76RLO 1830
}

Printed in the United States of America

Available to DOE and DOE contractors from the

Office of Scientific and Technical Information. P.O. Box 62, Oak Ridge, IN 37837; prices available from $(6,15)$ 576-8401.

Available to the public from the National Technical Information Service, U.S. Department of Commerce, 5285 Port Roval Rd., Springfield, VA 22161 


\section{DISCLAIMER}

Portions of this document may be illegible in electronic image products. Images are produced from the best available original document. 
PNL-10659

UC-605

STATISTICAL METHODOLOGY USED IN ANALYSES OF DATA FROM DOE EXPERIMENTAL ANIMAL STUDIES

E.S. Gilbert, Pacific Northwest Laboratory

W.C. Griffith, Inhalation Toxicology Research Institute

B.A. Carnes, Argonne National Laboratory

July 1995

Prepared for the

U.S. Department of Energy

under Contract DE-AC06-76RLO 1830

Pacific Northwest Laboratory

Richland, Washington 99352 



\section{SUMMARY}

This document describes many of the statistical approaches that are being used to analyze data from life-span animal studies conducted under the Department of Energy experimental radiobiology program. The methods, which are intended to be as informative as possible for assessing human health risks, account for time-related factors and competing risks, and are reasonably comparable to methods used for analyzing data from human epidemiologic studies of persons exposed to radiation

The methods described in this report model the hazard, or age-specific risk, as a function of dose and other factors such as dose rate, age at risk, and time since exposure. Both models in which the radiation risk is expressed relative to the baseline risk and models in which this risk is expressed in absolute terms are formulated. Both parametric and non-parametric models for baseline risks are considered, and several dose-response functions are suggested.

Tumors in animals are not always the cause of death but instead may be found incidentally to death from other causes. This report gives detailed attention to the context of observation of tumors, and emphasizes an approach that makes use of information provided by the pathologist on whether tumors are fatal or incidental. Special cases are those in which all tumors are observed in a fatal context or in which all tumors are observed in an incidental context.

Maximum likelihood theory provides the basis for fitting the suggested models and for making statistical inferences regarding parameters of these models. Approaches in which observations are grouped by intervals of time and possibly other factors are emphasized. This approach is based on iteratively reweighted least squares and uses Poisson weights for tumors considered to be fatal and binomial weights for tumors considered to be incidental. 


\section{CONTENTS}

SUMMARY ...........................

1.0 INTRODUCTION . . . . . . . . . . . . . . . 1.1

2.0 STATISTICAL MODELS $\ldots \ldots \ldots \ldots . \ldots \ldots$

3.0 CONTEXT OF OBSERVATION . . . . . . . . . . . . 3.1

4.0 FITTING THE MODELS . . . . . . . . . . . . 4.1

4.1 FITTING MODELS WHEN ALL TUMORS ARE FATAL . . . . . . . 4.1

4.2 FITTING MODELS WHEN ALL TUMORS ARE INCIDENTAL . . . . . 4.2

4.3 FITTING MODELS WITH BOTH FATAL AND INCIDENTAL TUMORS . . . 4.3

5.0 UNCERTAINTY IN ESTIMATED PARAMETERS . . . . . . . . . 5.1

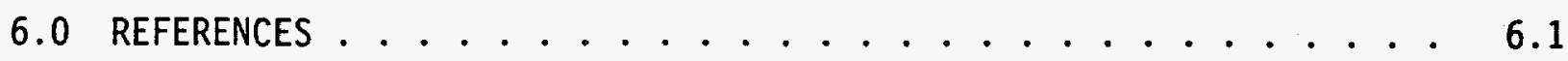




\subsection{INTRODUCTION}

Statisticians at Pacific Northwest Laboratory, the Inhalation Toxicology Research Institute, and Argonne National laboratory are collaborating in applying statistical methods that are as unbiased and informative as possible to past and current data from life-span animal studies conducted under the Department of Energy experimental radiobiology program. Emphasis is on studies in beagles, but data on selected studies in rodents are also evaluated. Analyses include both "standardized" and "combined" analyses. In standardized analyses, estimates of risk that are comparable across studies and across exposure groups are obtained for the most important life-span studies. In combined analyses, more extensive evaluations of specific questions of interest are made based on combined data from different laboratories and experiments.

The purpose of this document is to provide a detailed description of some of the statistical approaches that are being used in these analyses. Although the document is not intended to cover all methods that are being applied, it describes the most common approaches, especially those that are somewhat unique to these data, and, thus, have not been described in detail elsewhere. It is expected that details regarding specific applications of the methods described here will be given in papers and other documents describing results of these applications, including a document describing standardized analyses.

Because the purpose of the experimental studies is primarily to provide information for assessing human risks, methods are intended to be reasonably comparable to methods used in analyzing data from epidemiologic studies of humans exposed to radiation. These epidemiologic analyses have modeled the hazard, or age-specific death or incidence rates, as a function of dose ${ }^{1}$, and have also evaluated the modifying effects of factors such as dose rate, age at risk, and time since exposure. This approach has the advantage that it accounts for time-related factors and competing risks.

\footnotetext{
"Throughout this document, we use the word "dose", although "exposure" may be a more appropriate term for some applications.
} 
In some cases, certain aspects of experimental data require modification of methods used for human studies. First, baseline risks are often more uncertain for experimental animals than for humans. Human study populations frequently include large numbers of subjects with minimal exposure, and vital statistics provide additional information on baseline risks. The baseline lifetime risk for some types of cancer of interest in dogs and rodents may be very smal1, and available data on control animals are not always adequate for reliable estimation of the age-specific baseline risks needed for hazard modeling. For this reason, it is desirable in certain cases to avoid expressing risks relative to the baseline, as is often done in epidemiologic studies.

Human epidemiologic studies of cancer risks are often based on mortality data. In such studies, only cancers considered to be the cause of death are included, and the time of occurrence is usually defined as the date of death. Alternatively, human studies may be based on incidence data; in this case, the time of occurrence is usually defined as the date of diagnosis, but the statistical treatment is the same as in a mortality study.

By contrast, in animal studies, detailed examination of tissues is usually conducted after death. Thus, for certain tumors in certain species, a second important difference in human and animal data is that many tumors are identified that are not the cause of death, but are instead found incidentally to death from other causes. This is true, for example, for lung tumors in rats (Cross 1989, Chmelevsky et al. 1984, Gray et al. 1986).

The hazard model includes a variable indicating the time (or age) that the tumor occurred, and fitting this model requires appropriate use of available information on the time of death, and on whether or not the tumor was judged to have killed the animal or was an incidental finding. The correct approach to modeling is different if tumors are the cause of death than if they are incidental findings, and making the correct choice is especially important if competing risks depend on dose and other factors, or if time-dependent factors are of interest. 


\subsection{STATISTICAL MODELS}

We start by briefly reviewing the models used in recent analyses of data from epidemiologic studies of persons exposed to radiation, particularly those utilized used by the BEIR IV (1988) and BEIR V (1990) committees. A model in which the relative risk is assumed to be a linear function of exposure was emphasized in these analyses. The simplest linear relative risk model is one in which the hazard is given by $\lambda_{a}\left(1+\beta d_{a}\right)$, where $\lambda_{a}$ is the baseline risk at age $a$, and $d_{a}$ is a measure of dose at age a (possibly cumulative dose at some specified lag period before age $a)$, and $B$ is the excess relative risk.

A more general relative risk model for the hazard is as follows:

$$
h_{i k}\left(t, d_{t}\right)=\lambda_{t i}\left[1+F_{k}\left(d_{t}\right)\right]
$$

where $t$ is a measure of time (possibly age or a function of age), $\lambda_{t i}$ is the baseline risk at time $t, d_{t}$ is a measure of dose at age $t$ (again, possibly cumulative dose at some specified lag period before time $t), F_{k}$ is a function of dose and usually includes parameters to be estimated, and the subscripts $i$ and $k$ are used to indicate dependence of the baseline risks and the parameters of $F$ on factors such as type of exposure, sex, and laboratory. Separate subscripts $i$ and $k$ are used because baseline risks and the dose-response function may depend on different factors; however, this usage is not intended to exclude the possibility that some factors may affect both. For convenience, when no confusion is likely to result, we sometimes suppress the subscripts $i$ and $k$ by writing $h\left(t, d_{t}\right)$; occasionally we also write simply $h(t)$. The cumulative hazard is $H\left(t, d_{t}\right)=\int^{t} h\left(u, d_{u}\right) d u$.

In many epidemiologic applications (NAS 1988, NAS 1990, Lubin et a7. 1994), analyses have been based on a model in which the baseline risk was allowed to be arbitrary with separate parameters $\lambda_{\mathrm{ti}}$ estimated for each combination of $t$ and $i$. This approach has worked well because when tumors are the cause of death, the parameter $B$ can be estimated without explicit consideration of the parameters $\lambda_{t i}$, which cannot be estimated precisely. External rates, based on vital statistics, have also been used in modeling epidemiologic data.

For analyses that include tumors that are not the cause of death but are incidental tumors, analyses are greatly facilitated if a parametric baseline is used, leading to the following hazard 


$$
h_{k i}\left(t, d_{t}\right)=g_{i}(t)\left[1+F_{k}\left(d_{t}\right)\right]
$$

The Weibull function, $g_{i}(t)=\phi_{i} t^{\alpha}$, has been found to provide a good fit to many experimental animal data sets, and is thus a common choice. Other alternatives are the Gompertz function, $g_{i}(t)=\phi_{i} \exp (\alpha t)$, or a combined Weibull-Gompertz function of the form $g_{i}(t)=\phi_{i} \exp \left(\alpha_{1} t\right) t^{\alpha 2}$.

As noted in the introduction, the number of control animals is sometimes inadequate for obtaining precise estimates of the age-specific baseline risks, and thus estimates of risks relative to the baseline may be more uncertain than estimates of the absolute risk. Thus, it is sometimes desirable to express risk in absolute terms rather than relative to the baseline. A model that has been found to be very useful for this purpose is the following,

$$
h_{i k}\left(t, d_{t}\right)=(\alpha+1) t^{\alpha}\left[\lambda_{i}+F_{k}\left(d_{t}\right)\right]
$$

where $t$ is age (scaled as indicated below), $i, k, d_{t}$, and $F_{k}$ are defined as above, and $\lambda_{i}$ is the baseline risk.

By using an appropriate scaling of the age variable $t$ in equation (3), the coefficients $\lambda_{i}$ and the function $F_{k}(d)$ can be roughiy interpreted as lifetime risks, at least at low doses. It is easily shown that the cumulative hazard $H(t)$ for the excess risk due to a dose $d$ received at time $t=t_{E}$ is given by $F_{k}(d)\left[t^{\alpha+1}-t_{\mathrm{E}}^{\alpha+1}\right]$. If $t$ is scaled as a fraction of the age that is judged to correspond to an average life-span, the cumulative hazard to this average life-span is given by $F(d)\left[1-t_{E}{ }^{\alpha}\right]$, and for exposure received at birth, this becomes just $F(d)$. The probability of the endpoint being evaluated occurring by time $t$ is given by $1-\exp \{-H(t, d)\}$, which is approximately equal to $H(t, d)$ at small values of $H(t, d)$. Thus, with the parametrization given in (3), with age scaled as a fraction of the average life-span, and for the small doses of primary interest for risk assessment, the expression $F(d)$ can be regarded as an estimate of the lifetime risk associated with exposure at birth.

It can also be shown that if the factor $(\alpha+1)$ in equation (3) is replaced by $(\alpha+2)$, if $t$ is scaled as indicated above, and $F_{k}(d)$ is a linear function of the form $\beta_{k} d$, then $\beta_{k} d$ can be regarded as an estimate of the lifetime risk associated with lifetime exposure received at a constant exposure-rate. This parametrization has proved useful for analyzing experimental data on radon (Gilbert 1989). 
If a function other than the Weibull function is used for $g_{i}(t)$, it may be somewhat more difficult to evaluate lifetime risk. However, this can generally be accomplished by using numerical integration to evaluate $\int^{t} g_{i}(u) d u$, and the cumulative hazard $H(t)$. Regardless of the form of $g_{i}(t)$, alternative estimates of lifetime risk, taking into account the pattern of exposure over time, and the variation in survival, can be obtained if desired using lifetable methods.

A minimal latency period can be accounted for by including a "lag" time. For dogs, we have often defined $t=(a-365) /(13 * 365)$, where a indicates the age of the animal in days. In this case, there is a "lag" of 365 days or one year, and $13 * 365$ is the approximate average lifespan after the substraction of 365 days. For rats we have often defined $t=(a-60) / 720$. That is, a lag of 60 days has been assumed, and the average survival times has been assumed to be 780 days, or 720 days after the subtraction of the 60-day 1 ag. The determination of the average lifespan has been based on data from control animals.

Examples of dose-response functions that are commonly fit are linearquadratic functions of the form $F(d)=B_{1} d+B_{2} d^{2}$, and power functions of the form $F(d)=B d^{r}$. Both models allow examination and comparisons of the shape of the dose-response function, and allow tests for departures from linearity. With the linear-quadratic function, the linear component is of special interest because this component dominates at low doses.

Another dose-response function of interest is $F(d)=B_{j}$ (or $F(d)=\beta_{j} d$ ), where $j$ indexes categories defined by exposure. This form yields separate estimates of risk for each of several exposure categories, and is especially useful for comparison with models based on parametric dose-response functions. In analyzing tumors that are rare in control animals, estimates of the parameters $B_{j}$ may be very unstable if expressed relative to the baseline as in equations (1) and (2); thus, expressions of these parameters in absolute terms (as in equation (3)) may be especially desirable. It is noted that for these grouped analyses, common baseline parameters can be fit for all exposure groups within an experiment, and this requires that the parameters $B_{j}$ be fit simultaneously within each experiment.

The subscript $k$ in the models shown in equations (1), (2), and (3) allows for the possibility of addressing the modifying effects of factors such 
as dose rate, sex, exposure route and other factors. With the power model, for example, one could obtain separate estimates of $B$ and $\tau$ for males and females, and test whether or not this significantly improves the fit of the model. For some potential modifying factors, such as dose rate or time since exposure, it may be desirable to introduce parameters that measure the dependency on quantitative measures of the factor rather than simply obtaining separate estimates for various groups defined by the factor. For example, human data on exposure to radon have been analyzed using models of the form $F(d, z)=\beta d z^{\theta}$ and $F(d, z)=\beta d \exp (\theta z)$, where $z$ indicates variables such as exposure rate or exposure duration (Lubin et a7. 1994).

Analyses of human epidemiologic data on miners exposed to radon and radon progeny (NAS 1988, Lubin et a7. 1994) and on persons exposed to external radiation (NAS 1990) have examined dependencies of the risk relative to the baseline for various cancers on time since exposure, age at exposure, and age at risk. Because of limitations in the human data used to evaluate these dependencies, it is of interest to examine these dependencies using experimental animal data. In theory at least, this can be accomplished by introducing variables and parameters that measure these effects in modeling the hazard or age-specific risk. However, these variables may be time dependent, and may be highly correlated or even confounded with one another, making it difficult to estimate the individual effects of these factors.

Some special difficulties in assessing time-dependent factors arise in analyzing experimental data. For those cases where tumors are primarily incidental findings, it is known only that the tumor developed at a time prior to when it was observed, and thus less information is available on the time of occurrence than in cases where tumors are rapidly fatal. An additional difficulty is that for internal exposures to $\alpha$-emitting radionuclides, the distribution of dose over time is not known precisely.

There are also difficulties in addressing time-related factors in the same way that has been done in human studies, where such effects have been expressed in terms of the risk relative to the baseline risk. Because the pattern of baseline risks over time is often unknown or very uncertain for experimental animals, it may not be possible to address the departures from a constant relative risk model in the same manner as that used in analyzing human epidemiologic data. 


\subsection{CONTEXT OF OBSERVATION}

As noted above, lung tumors in rats are often not the cause of death, but are instead found incidentally to death from other causes. In order to make appropriate inferences about the hazard function, it is necessary to consider whether tumors are observed in a fatal or incidental context.

Peto et a7. (1980) propose guidelines for testing for dose-response relations using information provided by the pathologist on whether tumors were observed in the incidental or fatal context. Mcknight and Crowley (1984) provide a mathematical model for addressing this problem, and examine conditions under which various approaches are appropriate. They find that without adequate sacrifice data, unbiased inferences can be made only if additional assumptions are made.

In this document, the approach emphasized is to use information provided by the pathologist on whether tumors are fatal or incidental. This general approach has been recommended by Peto et al. (1980) for testing purposes, and expanded by Peto et al. (1991), Gart et al. (1986), Crump (1992), and Dewanji et al. (1993) for the more general purpose of making inferences about the hazard function. Mcknight and Crowley show that this approach is appropriate if the probability of dying from a cause other than the tumor of interest is unaffected by the presence of a tumor, and if the determination of cause of death has been made correctly. Neither of these assumptions are likely to be satisfied exactly, but, in the absence of a large amount of sacrifice data, making some additional assumptions cannot be avoided. The sensitivity of analyses to the classification of tumors as fatal or incidental can be examined by considering alternative classifications.

Gart et al. (1986) propose a general model for combining fatal and incidental tumors that involves the specification of two separate hazard functions: $h(t)$ based on the time that the tumor becomes detectable at necropsy, and $h_{F}(t)$ based on the time that the tumor kills the animal. They define $Q(t)$ as the conditional probability of tumor onset after time $t$, given tumor-free survival through time $t$, and note that $Q(t)=S(t) / S_{F}(t)$, where $S(t)$ and $S_{F}(t)$ are the survival functions associated with the respective hazard functions $h(t)$ and $h_{F}(t)$; that is, $S(t)=\exp \{-H(t)\}, S_{F}(t)=\exp \{-$ $\left.H_{F}(t)\right\}$, and thus $Q(t)=\exp \left\{-\left[H(t)-H_{F}(t)\right]\right\}$. Gart et al. further note that 
the likelihood can then be separated into a component similar to that used for analyses where all tumors are considered as fatal, and one that is similar to that where a11 tumors are considered as incidental with $H(t)-H_{F}(t)$ playing the role of the cumulative hazard. Gart et al. also note that non-parametric estimates of the survivor function can be obtained using methods described by Kodell et al. (1982), provided $Q(t)$ is monotonically decreasing.

Peto et al. (1991) and Dewanji et al. (1993) have used an approach that is a special case of that indicated above. Specifically, they make the assumption that $h_{F}(t)=f h(t)$, or that the hazard function for a fatal tumor is a fixed fraction $f$ of the hazard for an incidental tumor. A more general model, which we have found useful, can be based on the assumption $h_{F}(t)=f t^{\alpha^{*}}$ $h(t)$. Note that for the Weibull model given in equation (3),

$$
h_{F}(t)=(\alpha+1)\left(f^{*} t^{\alpha^{*}}\right)^{\alpha}\left[\lambda+F\left(d_{t}\right)\right]
$$

where $f^{*}=f^{1 / \alpha}$, and thus, with this model, the time a tumor becomes observable at necropsy is a fraction $f^{*} t^{\alpha^{*}}$ of the time that the tumor kills the animal. In applying this more general model, one must take care that $\alpha^{\star}$ is amall enough that $f t^{\alpha^{*}}$ does not exceed 1 for reasonable values of $t$.

Statistical methods that do not require pathologists' judgments about individual tumors, but are instead based on general assumptions about the lethality of tumors, have also been proposed. These assumptions may be specified, for example, in terms of the relationship of the hazards for the occurrence of tumors and the occurrence of fatal tumors, or in terms of the distribution of the time required for tumors to kill animals; assumptions about the hazard for mortality from other causes may also be required. Such methods are likely to be especially valuable when judgments regarding the context of observation of tumors are unavailable, or are considered inadequate, and may also prove useful in examining the sensitivity of results to assumptions about tumor lethality. 


\subsection{FITTING THE MODELS}

The approach to fitting the hazard models described above, and to making inferences about the parameters of these models, is based on maximum likelihood theory; this theory is described in numerous statistical textbooks including Kalbfleish and Prentịce (1980), and Cox and Hinkley (1974). We first describe the approach used to fit the hazard model in the situations where either all tumors are assumed to be fatal or all tumors are assumed to be incidental; these are referred to as "fatal" and "incidental" analyses, respectively.

\subsection{FITTING MODELS WHEN ALL TUMORS ARE FATAL}

Breslow and Day (1987) describe methods for fitting hazard models based on either grouped or continuous data, emphasizing the flexibility and efficiency in using grouped data. Below, the use of data in which observations are grouped by intervals of time is emphasized with a constant hazard function assumed for each time interval. A continuous approach could also be used for many of the models under consideration, especially those in which exposure does not change with time. Initially, the approach is described with no further grouping of data by categories of dose and other variables.

Using this grouped approach, fatal analyses are based on "dog-years" or "rat-days", comparable to the person-year approach often employed in epidemiologic studies. Constructing the data set to be used for such analyses involves constructing a data set including an observation for each of several specified intervals in each dog's or rat's lifespan. Often, one-year intervals have been used for dogs and 60-day intervals have been used for rats, and one-year or 60-day lags have also been incorporated. Each of these observations is assigned a value $t$, the scaled age in the middle of the preceding interval (for a single interval lag), a dose $d_{t}$, the cumulative dose up to the middle of the preceding interval, and an indicator variable $Y_{t}$ such that $Y_{t}=1$ if the animal died of a tumor of the type of interest in interval $t$, and $Y_{t}=0$ otherwise. The expected value of $Y_{t}$ is given by expressions for the hazard function (as given in equations (1), (2), or (3)) multiplied by the length of the interval. That is, 


$$
E\left(Y_{t}\right)=L_{t} h\left(t, d_{t}\right)
$$

were $L_{t}$ is the length of interval $t$, and $h\left(t, d_{t}\right)$ is the hazard for age $t$, dose $d_{t}$. Maximum likelihood estimates of the parameters of the hazard function can be obtained using iteratively reweighted least squares, treating the $Y_{t}$ as Poisson variables.

To facilitate analyses, especially in experiments with large numbers of

animals, doses can be grouped into several categories, and files of dog-years or rat-days collapsed into a smaller file such that each observation corresponds to a category defined by time, dose category, and possibly other factors. Provided a sufficient number of dose categories are used to cover the dose range adequately, there is little loss of information using this approach. This can be verified by refitting the models judged most important using data that are not grouped on dose.

Several software packages are available for implementing these analyses with a parametric baseline including the SAS (1985) NLIN procedure. The AMFIT module of the software package EPICURE (Preston et al. 1993) allows flexible modeling with both parametric and non-parametric baseline functions.

\subsection{FITTING MODELS WHEN ALL TUMORS ARE INCIDENTAL}

For incidental analyses, a data set is constructed with an observation for each animal, and includes a variable $Y$ indicating whether or not the animal has a tumor of the type of interest, and also includes a vector with information on dose for each time period (single years for dogs; 60 days for rats) in the animal's lifespan, comparable to the information used in fatal analyses. Other variables such as sex and dose rate may also be included. In this case, the expected value of $Y$ is the cumulative probability (denoted by $P$ ) of developing a tumor by the time of death $t . P$ is related to the hazard $h\left(t, d_{t}\right)$ by $P=1-\exp \left\{-H\left(t, d_{t}\right)\right\}$, where $H\left(t, d_{t}\right)=\int^{t} h\left(u, d_{u}\right)$ du is the cumulative hazard.

The cumulative hazard can be approximated by sums of terms of the type defined by equation (4). That is,

$$
E(Y)=1-\exp \left\{-\Sigma_{u} L_{u} h\left(t, d_{u}\right)\right\}
$$

Maximum likelihood estimates can be obtained using iteratively reweighted least squares, with the $Y$ treated as binomial variables with expectation $P$. 
As in fatal analyses, data can be grouped for animals in the same time and dose category at death, and incurring the same pattern of exposure over time.

\subsection{FITTING MODELS WITH BOTH FATAL AND INCIDENTAL TUMORS}

As discussed above, one approach for analyses including both fatal and incidental tumors is to model two separate hazard functions, $h(t)$ and $h_{F}(t)$. With this formulation, terms are contributed to the likelihood function as follows. For an animal with a fatal tumor (regardless of whether the animal also has incidental tumors), the term contributed indicates the likelihood that the animal remained free of a fatal tumor until time $t$, but died of a fatal tumor at time $t$, and is given by $h_{F}(t) \exp \left\{-H_{F}(t)\right\}$. For an animal with an incidental tumor but no fatal tumors, the term contributed indicates the likelihood that the animal developed a tumor by time $t$, less the likelihood that the animal died of a tumor by time $t$, and (using the notation of Gart et a7. 1986) is given by $Q(t)=\exp \left\{-\left[H(t)-H_{F}(t)\right]\right\}$. For an animal with no tumors, the term contributed indicates the likelihood that the animal remained tumor free until time $t$, and is given by $\exp \{-H(t)\}$.

Let $j$ index animals in the study, and let $h_{j}(t)$ and $h_{F j}(t)$ denote hazards for tumor incidence and mortality, respectively, for animal $j$. Let $\mathrm{YF}_{j}$ equal 1 if an animal has a fatal tumor, and 0 otherwise; and let $Y I_{j}$ equal 1 if an animal has at least one incidental tumor but no fatal tumor, and 0 otherwise. The likelihood function can then be written as the product over all animals of the terms

$$
\begin{aligned}
\left\{h_{F j}(t)\right\}^{Y F j} \exp \left\{-H_{F j}(t)\right\}[ & {\left[1-\exp \left\{-\left[H_{j}(t)-H_{F j}(t)\right\}\right]^{\mathrm{YIj}}\right.} \\
& \times\left[\exp \left\{-\left[H_{j}(t)-H_{F j}(t)\right]\right\}^{(1-r i j)} .\right.
\end{aligned}
$$

Note that if all tumors are instantly fatal, then $H_{F}(t)=H(t)$ and expression (6) reduces to the usual likelihood for "fatal" analyses. Alternatively, if tumors are never fatal, then $H_{F}(t)=0$ and the expression (6) reduces to the likelihood for "incidental" analyses.

In general, the first portion of this likelihood, $\left\{h_{F j}(t)\right\}^{\mathrm{rFj}} \exp \left\{H_{F j}(t)\right\}$, is of the form that one would use for "fatal" analyses if only fatal tumors were considered, while the remaining portion of the likelihood is of the form that one would use for "incidental" analyses if animals with fatal tumors were first removed. Analyses combining fatal and incidental tumors can thus be implemented by combining two files of observations: the first of the type 
used for "fatal" analyses, including all animals but only fatal tumors, and the second of the type used for "incidental" analyses but excluding animals with fatal tumors. Maximum likelihood estimates can then be obtained using iteratively reweighted least squares with Poisson weights for observations from the "fatal" file, and binomial weights for observations from the "incidental" file. The SAS (1985) procedure NLIN can be used to carry out the necessary computations.

It may be of interest to note that in the special case where $h_{F}(t)=f h(t)$, the expression in equation $(6)$ becomes

$$
\begin{aligned}
\left\{f h_{j}(t)\right\}^{\mathrm{YFj}} \exp \left\{-f H_{j}(t)\right\} & {\left[1-\exp \left\{-(1-f) H_{j}(t)\right\}\right]^{\mathrm{YIj}} } \\
\times & {\left[\exp \left\{-(1-f) H_{j}(t)\right\}\right]^{(1-Y i j)} . }
\end{aligned}
$$

Crump et al. (1992) fitted models using the following likelihood for analyzing data with both fatal and incidental tumors:

$$
h_{j}(t)^{\gamma F j} \exp \left\{-H_{j}(t)\right\}\left[1-\exp \left\{-H_{j}(t)\right\}\right]^{r I j}\left[\exp \left\{-H_{j}(t)\right\}\right]^{\left(1-Y_{I j}\right)} .
$$

This is of the same general form as that in equation (7) with $f=0.5$. Because the Crump et al. likelihood does not include the factor 0.5 , the hazard estimated is that associated with death from tumor $\left\{h_{F}(t)\right\}$, which is half the value of the hazard for tumor occurrence, estimated using the expression in (7). 


\subsection{UNCERTAINTY IN ESTIMATED PARAMETERS}

There are several maximum likelihood-based approaches for expressing uncertainty in parameters. Asymptotically, these approaches yield equivalent results, but with modest sample sizes, some approaches are to be preferred over others.

Software packages that are used to fit these models, such as the SAS (1985) parameters, and these can be used to express uncertainty and to obtain confidence intervals. However, for some models and parameters, such as the coefficients of dose in models where $F(d)$ is a linear-quadratic or power function of dose, these asymptotic standard errors may not provide an adequate assessment of the uncertainty (Prentice and Mason 1986). An alternative procedure is to estimate standard errors for the logarithms of the coefficients, and then express uncertainty using multiplicative or geometric standard errors. Likelihood ratio based confidence limits may also be calculated; this approach is more difficult to implement, but may be valuable for obtaining upper confidence limits when estimated coefficients are zero or negative, and the multiplicative approach does not work. This is especially likely to occur for lower dose categories in grouped analyses, for the linear coefficient in the linear-quadratic model, or for the coefficient in a linear model restricted to smaller doses. The improvement in fit resulting from the addition of parameters to the model can be assessed using likelihood ratiobased tests. 


\subsection{REFERENCES}

Breslow, N.E. and N.E. Day. 1987. Statistical Methods in Cancer Research: Volume II -- The Design and Analys is of Cohort Studies. IARC Scientific Publications No. 82. Lyon, France: International Agency for Research on Cancer.

Chmelevsky, D., A.M. Kellerer, J. Lafuma, and J. Chameaud. 1984. "Maximum likelihood estimation of the prevalence of nonlethal neoplasms -- an application to radon-daughter inhalation studies." Radiat. Res. 98:519-535.

Cox, D.R., and D.V. Hinkley. 1974. Theoretical statistics. London: Chapman and $\mathrm{Hall}$.

Cross, F.T. 1989. "Evidence of lung cancer risk from animal studies." In: Proceedings of the Twenty-fourth Annual Meeting of the NCRP, Naomi H. Harley, ed. March 30-31, 1988, Bethesda MD: National Council on Radiation Protection and Measurements.

Crump, K.S., R.B. Howe, C. Van Landingham, and W.G. Fuller. 1992. TOX RISK, Version 3.1, TOXicology RISK Assessment Program. Ruston, LA: Clement International Corporation.

Dewanji, A., D.Krewski, and M.J. Goddard. 1993. "A Weibull model for the estimation of tumorigenic potency." Biometrics 49:367-377.

Gart, J.J., D. Krewski, P.N. Lee, R.E. Tarone, and J. Wahrendorf. 1986. Statistical Methods in Cancer Research: Volume III -- The Design and Analysis of Long-Term Animal Experiments. IARC Scientific Publications No. 79. Lyon, France: International Agency for Research on Cancer, Lyon, France.

Gilbert, E.S. 1989. "Lung cancer risk models from experimental animals." In: Proceedings of the Twenty-fourth Annual Meeting of the NCRP, Naomi $H$. Harley, ed., pp. 141-155. Bethesda MD: National Council of Radiation Protection and Measurements.

Gray, R.G., J. Lafuma, S.E. Parish, and R. Peto. 1986. "Lung tumors and radon inhalation in over 2000 rats: approximate linearity across a wide range of doses and potentiation by tobacco smoke." In: Lifespan Radiation Effects Studies in Animals: What can they tell us? Proceedings of the twenty-second Hanford Life Sciences Symposium. Available from National Technical Information Service, U.S. Department of Commerce: pp. 455-470 No. DE87000490 (CONF-830951).

Kalbfleisch, J.D. and R.L. Prentice. 1980. The Statistical Analysis of Failure Time Data. New York: John Wiley and Sons.

Kode11, R.L., J.H. Farmer, D.W. Gaylor, A.M. Cameron. 1982. "Influence of cause-of-death assignment on time-to-tumor analyses in animal carcinogenesis studies." J Natl Cancer Inst 69:659-664. 
Lubin, J.H., J.D. Boice, Jr., C. Edling, R.W. Hornung, G. Howe, E. Kunz, R.A. Kusiak, H.I Morrison, E.P Radford, J.M. Samet, M. Tirmarche, M., A. Woodward, S.X. Yao, and D.A. Pierce. 1994. Radon and Lung Cancer Risk: A Joint Analysis of 11 Underground Miners Studies. National Institutes of Health, National Cancer Institute. NIH Publication No. 94-3644. Washington, D.C.: U.S. Department of Health and Human Services.

Mcknight, B. and J. Crowley. 1984. "Tests for differences in tumor incidence based on animal carcinogenesis experiments." J. Am. Statist. Assoc. 79:639648.

National Academy of Sciences. 1988. Health Risks of Radon and Other Internally Deposited Alpha-Emitters. BEIR IV. Washington D.C.: National Academy Press.

National Academy of Sciences. 1990. Health Effects of Exposure to Low Levels of Ionizing Radiation. BEIR V. Report of the Committee on the Biological Effects of Ionizing Radiations, National Research Council. Washington, D.C.: National Academy of Sciences.

Peto, R., N. Day, R. Gray, P. Lee, S. Parish, J. Peto, S. Richards, and H. Wahrendorf. 1980. "Guidelines for simple, sensitive significance tests for carcinogenic effects in long-term animal experiments." In IARC monographs on the evaluation of the carcinogenic risk of chemicals to human. Supplement 2 , Long-term and short-term screening assays for carcinogens: a critical appraisal, pp. 311-346. Lyon, France: International Agency for Research on Cancer.

Peto, R., R. Gray, P. Brantom, and P. Grasso. 1991. "Dose and time relationships for tumor induction in the liver and esophagus of 4080 inbred rats by chronic ingestion of $\mathrm{N}$-Nitrosodiethylamine or $\mathrm{N}$-Nitrosodimethylamine." Cancer Research 51:6452-6469.

Prentice, R.L. and M.W. Mason. 1986. "On the application of linear relative risk regression models." Biometrics 42:109-20.

Preston, D.L., J.H. Lubin, and D.A. Pierce. 1993. Epicure User's Guide. Seattle: HiroSoft International Corp.

SAS Institute Inc. 1985. SAS User's Guide: Statistics, Version 5 Edition. Cary, NC: SAS Institute Inc. 


\section{DISTRIBUTION}

Number

of Copies

\section{$\underline{\text { OFFSITE }}$}

12 DOE/Office of Scientific and

Technical Information

M.G. Frazier

Office of Health and Environmental Research

ER-72/F209/GTN

U.S. Department of Energy

Washington, DC 20585

W.C. Giffith (5)

ITRI

P.0. Box 5890

Bldg. 9217 Area Y Kirtland

Air Force Base East

Albuquerque, NM 87185
Number

of Copies

B.A. Carnes (5)

Division of Medical and Biological Research Argonne National Laboratory 9700 S. Cass Ave B1dg. 202 Argonne, IL 60439

\section{ONSITE}

18 E.S. Gilbert (10)

P7 -82

J.A. Mahaffey

P7 -82

R.E. Weller

P7 -52

C.R. Watson

P7 -82
Publishing Coordination

Technical Information (5) 